\title{
Diversity in Chemical Engineering Education: Status and Perspectives
}

\section{Dr. Cheryl A Bodnar, University of Pittsburgh}

Cheryl A. Bodnar, PhD, CTDP is an Assistant Professor (Teaching Track) in the Department of Chemical and Petroleum Engineering at the Swanson School of Engineering at the University of Pittsburgh. She obtained her certification as a Training and Development Professional (CTDP) from the Canadian Society for Training and Development (CSTD) in 2010, providing her with a solid background in instructional design, facilitation and evaluation.

Dr. Bodnar's research interests relate to the incorporation of active learning techniques in undergraduate classes (problem based learning, games and simulations, etc.) as well as integration of innovation and entrepreneurship into the Chemical and Petroleum Engineering as well as broader engineering curriculum. In addition, she is actively engaged in the development of a variety of informal science education approaches with the goal of exciting and teaching K-12 students about regenerative medicine and its potential.

\section{Dr. Arthur Felse, Northwestern University \\ Dr. Karen A High, Oklahoma State University \\ Dr. Jason M. Keith, Mississippi State University}

Jason Keith is the Interim Dean and Earnest W. and Mary Ann Deavenport, Jr. Chair in the Bagley College of Engineering at Mississippi State University, a position he has held since March, 2014. Keith received his B.S. in Chemical Engineering from The University of Akron and his $\mathrm{PhD}$ from the University of Notre Dame. He was a faculty member at Michigan Technological University from 2000-2011 and was Director of the Dave C. Swalm School of Chemical Engineering and holder of the Earnest W. Deavenport Chair from 2011-2014. Keith received the Raymond W. Fahien Award from the Chemical Engineering Division of ASEE in 2008.

\section{Dr. Adrienne Minerick, Michigan Technological University}

Adrienne Minerick received her M.S. and Ph.D. from the University of Notre Dame and B.S. from Michigan Technological University. Adrienne's research interests include electrokinetics, predominantly dielectrophoretic characterizations of cells, and the development of biomedical microdevices. She earned a NSF CAREER award and was nominated for Michigan Professor of the Year in 2014. Research within her Medical micro-Device Engineering Research Laboratory (M.D. - ERL) also inspires the development of Desktop Experiment Modules (DEMos) for use in chemical engineering classrooms or as outreach activities in area schools (see www.mderl.org). Adrienne is currently co-Chair of ASEE's Diversity Committee and PIC I Chair; she has previously served on WIED, ChED, and NEE leadership teams and contributed to 37 ASEE conference proceedings articles.

\section{Dr. Ann Saterbak, Rice University \\ Dr. Jennifer Cole, Northwestern University}

Jennifer Cole is the Assistant Chair in Chemical and Biological Engineering in the Robert R. McCormick School of Engineering and Applied Science at Northwestern University. Dr. Cole's primary teaching is in capstone and freshman design, and her research interest are in engineering design education. 


\title{
Diversity in Chemical Engineering Education: Status and Perspectives
}

\begin{abstract}
For several years now, there has been a call for a more diverse workforce within engineering. In 1998, the National Academy of Engineering published a report titled "Diversity in Engineering" that described how the current state of the engineering workforce was not representative of the population of the United States. The report went on to outline that without representation of under-represented minorities, women as well as individual diversity, the solutions to engineering problems could suffer from a lack of input from individuals with different life experiences. ${ }^{1}$ Sixteen years later we find ourselves not significantly ahead of where we were at the time the report was issued. Recently, the National Science Foundation's biennial report on "Women, Minorities and Persons with Disabilities in Science and Engineering" was published which showed that the engineering and scientific workforce is still made up of $51 \%$ white males ${ }^{2}$ despite continued efforts on the part of academic institutions, professional organizations and other stakeholders to address this issue.
\end{abstract}

As part of the ASEE's "Year of Action on Diversity", the Chemical Engineering division assembled a committee to perform a review of the state of diversity within its division and to identify opportunities where improvements could be made and a plan for accomplishing these goals. The diversity committee performed preliminary analysis of the Chemical Engineering division's membership information and compared it against diversity data for engineering faculty and the overall engineering workforce. Chemical engineering divisions' membership information was obtained from ASEE's membership unit, data on diversity in engineering workforce was extracted from NSF's workforce data archive ${ }^{2}$, and data on diversity in engineering faculty was extracted from ASEE's Engineering by the Numbers document. ${ }^{3}$ It was found that gender diversity in the division exceeded that of both doctoral level professionals and chemical engineering faculty but still fell short of the general working population. Diversity of underrepresented minorities within the division was at similar levels to those within doctoral level professions and chemical engineering faculty except for the Asian/Pacific Islander group which was found to be underrepresented in the division. Gender diversity among doctoral students in chemical engineering is significantly low, though gender diversity at the bachelor's level is more favorable than in other engineering disciplines. There appears to be a bottleneck in translating the favorable gender diversity at the bachelor's level to doctoral level students.

In an effort to inform the division of the current status of diversity and provide a forum for change to occur, the division will host a diversity panel with panelists from different career stages that can share their experiences and perspectives on diversity. The panelists were each provided a survey with open-ended questions in order to obtain their perspectives on diversity within the field of chemical engineering and what needs to be done in order to improve upon it. Themes relating to better support structures, training on implicit bias and broadening the discussion beyond individuals that fall into under-represented categories to include the division as a whole were discussed. 


\section{Introduction}

As part of the ASEE's "Year of Action on Diversity", the Chemical Engineering division assembled a committee to perform a review of the state of diversity within its division and to identify opportunities where improvements could be made and a plan for accomplishing these goals. According to the ASEE website, diversity can include but is not limited to "age, belief system, disability status, ethnicity, gender, gender identity, gender expression, national origin, race, sexual orientation, socio-economic status, and any other visible or non-visible differences." We believe that this definition provides a great overview of what diversity is and how it occurs on multiple levels, many of which are not visible. With this definition in mind, the committee sought to create a mission for its committee over the three year time frame it will be in place. The mission statement for the committee is "we will promote an inclusive chemical engineering education community and empower underrepresented groups through informing the division membership about the state of diversity within the division, raising awareness of diversity issues being faced by its membership, establishing best practices for how to create a diverse and inclusive membership now and in the future, and providing resources and opportunities for women and underrepresented minorities to excel in the chemical engineering profession." The mission established should allow for more information to be disseminated to the division membership and create a supportive environment where members can learn from one another on how to address diversity issues in their work place and develop an inclusive learning and work environment.

\section{Historical Overview of Diversity Initiatives in Engineering Education}

Engineering education has a rich history in promoting diversity in both student and faculty groups. ASEE created a central diversity committee in the fiscal year 2011 with a strategic goal to increase diversity in engineering education. Most recently ASEE declared 2014-15 as the 'year of action on diversity' with a specific goal to engage individual divisions in diversity initiatives. The two primary divisions for diversity within ASEE -Women in Engineering Division and Minorities in Engineering Division adopted their first bylaws in 1978 and 1999 respectively. These divisions have since done a tremendous amount of work in promoting diversity in engineering education. Also, the Corporate Member Council, the International Division, and the Two-year College Division have made specific commitments to increase diversity within their groups. The Mechanical Engineering Division donated \$500.00 for ASEE's Diversity Best Paper award. However, at this point Chemical and Mechanical Engineering are the only two core engineering discipline divisions that have committed to a diversity effort. It is our hope that this will change during the 'year of action on diversity'.

To quantify the prevalence of diversity-related efforts in engineering education, we did a keyword search for articles that appeared in the Journal of Engineering Education (JEE) and in the European Journal of Engineering Education (EJEE). The number of relevant articles in these journals will reflect the diversity-related efforts happening in the United States (JEE) and Europe (EJEE). The keywords 'diversity', 'women', 'minority', and 'gender' were used as search terms for homologous hits in article titles. Two other keywords commonly associated with diversity 'inclusive', and 'race' were not used due to overlaps with other topic areas (such as car race). It should be noted that minor overlap between the search terms is possible, but screening was performed to minimize double counting. The papers were not weighted or scored based on their 
contribution to diversity. So in this study, a particular paper could have an extensive study on diversity and that would count as much as a simple study/discussion on diversity. We plan on performing a more detailed systematic analysis of the papers based on their contribution to diversity in the future.

Our analysis of keyword search data indicates that JEE and EJEE have published a similar number of articles related to diversity till now, approximately 50 and 56 papers respectively. Itemization of publication data based on a historical timeline reveals the extent of diversityrelated engineering education research during various time periods. The timeline data is presented in Table 1.

Table 1. Itemized timeline of diversity-related publications

\begin{tabular}{|l|c|c|}
\hline \multirow{2}{*}{ Time period } & \multicolumn{2}{|c|}{ Number of diversity related publications } \\
\cline { 2 - 3 } & JEE & EJEE \\
\hline $2010-2014$ & 8 & 17 \\
\hline $2005-2009$ & 7 & 22 \\
\hline $2000-2004$ & 13 & 4 \\
\hline $1995-1999$ & 16 & 0 \\
\hline$<1995$ & 6 & 13 \\
\hline Total & 50 & 56 \\
\hline
\end{tabular}

It is evident that in JEE (which for this work is being considered as representative of the United States) there was not a significant amount of diversity-related studies published until 1995, but publication of research in this area rapidly increased afterwards. The decade between 1995 and 2004 had the highest number of articles published on diversity, a total of 29 . However, the number of articles dropped in the following decade to a mere 15 (2004-2014). It is possible that this decrease that is observed is due to studies on diversity-related issues being targeted to other journal sources that are more relevant to this field. We plan on investigating the reasons for this drop in the future, but this trend certainly presents a matter of concern - especially since JEE is considered to be the premier engineering education journal within the United States and is also highly regarded internationally.

The European engineering education community (based on papers published in EJEE) has published a larger number of papers on diversity-related topics in the years before 1995 - a little over twice the number that was found within JEE. This was followed by a steep drop in the number of articles to 4 published studies in the decade between 1995 and 2004, unlike in the United States where the number of articles peaked at 29 during the same decade. Immediately after this drop a rapid increase to 22 publications in the 2005-2009 time period was observed which was followed by a slight decrease during the 2010-2014 time period. Data on the number of engineering education articles published within JEE and EJEE indicate that diversity-related studies in these publications may be occurring at a reduced rate or as mentioned previously that authors of this type of work are not targeting these specific journals as their means of dissemination. As these two journals are important reference sources for many within the engineering education field it is important to see continued representation of diversity work in these journals in the years ahead. 
To determine the extent of diversity-related education research in the chemical engineering discipline, we performed the same key word search in the journals Chemical Engineering Education (CEE) and Education for Chemical Engineers (ECE). We searched all articles in ECE from 2006, the year of its launch, until 2014. The CEE started publication in 1962, but it's not clear how far back the CEE keyword search extends - this information is not available on the CEE website. CEE and ECE published a total of 11 and 5 papers related to diversity until present. Due to the smaller number of papers in CEE and ECE, the data was not itemized based on timelines. Considering the fact that CEE and ECE published 34 and 10 papers respectively in 2014 alone, the fraction of diversity-related papers within these journals is low. Thus there is an immediate need to encourage submission of diversity-related studies pertinent to the chemical engineering field to these chemical engineering education journals.

\section{Status of Diversity in the Chemical Engineering Division}

Intent of the work described in this section are: (i) to quantitatively understand the gender and ethnic diversity in the Chemical Engineering division membership, (ii) to comparatively study the diversity pipeline from undergraduate to faculty levels in the chemical engineering and the overall engineering disciplines, (iii) propose areas that need to be addressed immediately in the chemical engineering discipline. Chemical Engineering divisions' membership information was obtained from the ASEE's membership unit ${ }^{5}$, data on diversity in engineering workforce was extracted from NSF's workforce data archive ${ }^{2}$, and data on diversity in engineering faculty was extracted from ASEE's Engineering by the Numbers document ${ }^{3}$. The Chemical Engineering division membership dataset reported 536 members, including 6 members who did not list their gender and 144 members who did not list their ethnicity. A distinction between members from academia and industry in the Chemical Engineering division is not made in this study because such information was not available at this time. It should be noted that the Chemical Engineering division has members from outside the United States, and they are included in the statistics presented in this paper. But the statistics for diversity in workforce is based on numbers in the United States only. As a part of future work, we plan on looking specifically at the Chemical Engineering division's diversity based on members in the United States to provide a direct comparison. Some percentage values reported may not add up to one hundred due to rounding.

Gender diversity within the Chemical Engineering division's membership exceeds diversity in chemical engineering faculty and doctoral level professionals (Figures $1 \mathrm{a}-\mathrm{c}$ ). Gender diversity among chemical engineering faculty is slightly better than the combined diversity in all engineering disciplines by $3.2 \%$. The percentage of female members in the Chemical Engineering division $(21.83 \%)$ is higher than their percentage in chemical engineering professorship as a whole (17.7\%), i.e., the Chemical Engineering division is enriched with female members relative to the chemical engineering faculty population. It is possible to hypothesize that a higher percentage of women might have careers as teaching faculty in research universities or work at undergraduate-focused universities - the populations that are most likely to be engaged with ASEE. Itemized data on chemical engineering faculty numbers in research- and undergraduate-focused universities is not readily available. We plan on testing this hypothesis in the future with a broader data search. Though gender diversity in Chemical Engineering division membership is favorable, the data does not necessarily capture all aspects of the chemical engineering professorship, particularly individuals working at research universities. Data on gender diversity at the bachelor's degree level indicates that chemical 
engineering undergraduate programs are significantly more diverse compared to the combined diversity in all engineering disciplines by $13.2 \%$ (Figure 1d). It is important to observe that chemical engineering gender diversity drops from $32.3 \%$ at the bachelor's level to $10 \%$ at the doctorate level. Thereafter gender diversity increases to $17.7 \%$ at the faculty level. Therefore, the bottleneck for gender diversity in chemical engineering education appears to be correlated to the doctoral level. Based on this data, we believe it is vital for the chemical engineering community to take efforts to recruit and retain higher number of women students in doctoral programs.

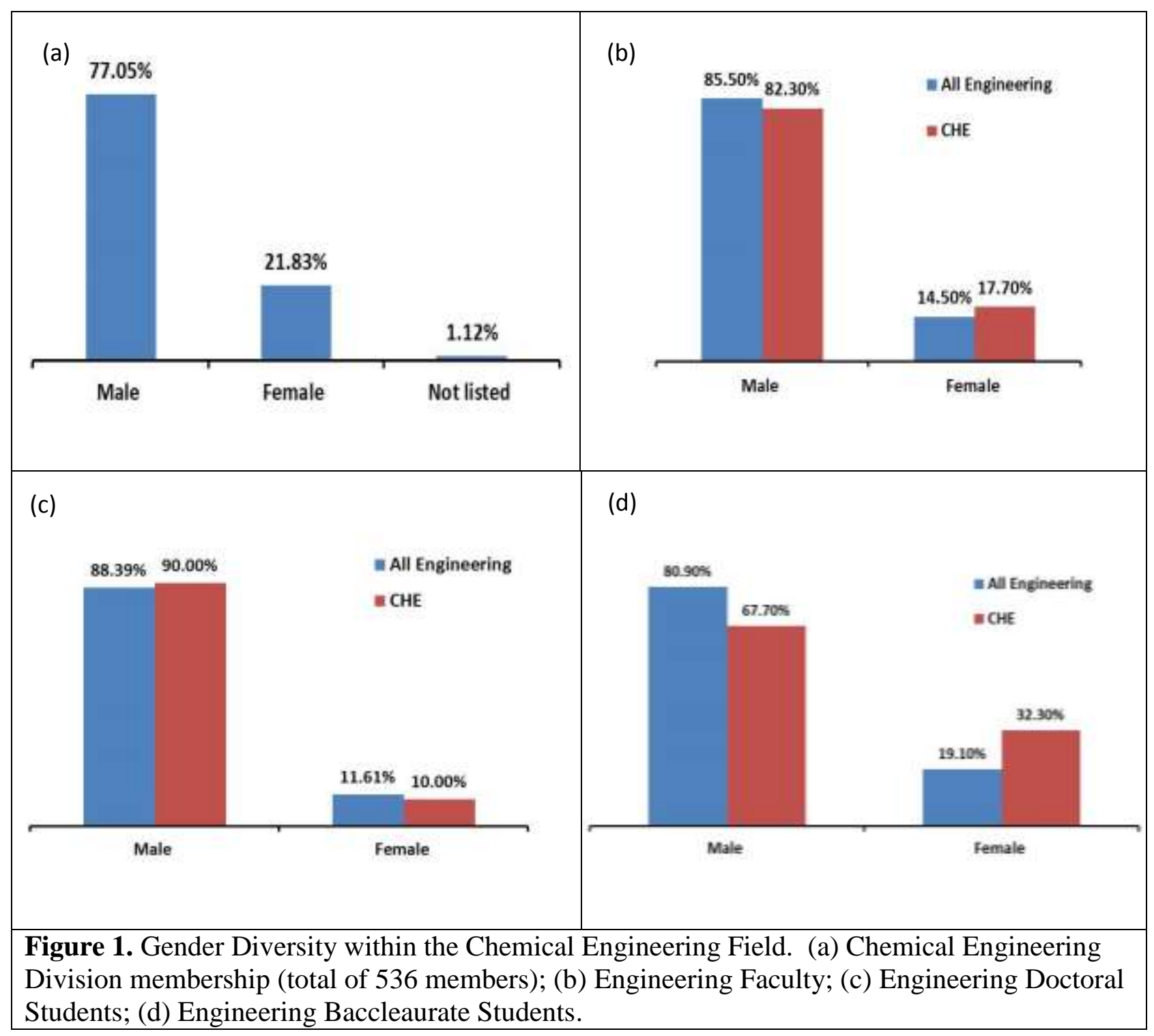

Even with some favorable diversity in chemical engineering education, women are still underrepresented when compared to the general working population in the United States which has $44.5 \%$ women. Chemical engineering education is also less diverse than the combined science and engineering occupations which have $27.5 \%$ women. Thus, besides the immediate need for gender diversity at the doctoral level, participation of women at all levels of chemical engineering education should be improved to make it proportionate to the general working population. 
Ethnic diversity in the Chemical Engineering division's membership is representative of diversity in chemical engineering faculty and doctoral level professionals, except for the Asian/Pacific Islander group which is underrepresented in the Chemical Engineering division membership - this group makes up $21.9 \%$ of faculty but only $14 \%$ of the division membership (Figures $2 \mathrm{a}, \mathrm{b}$ ). This trend is also true for all engineering. But, the Asian/Pacific Islander group is more underrepresented at the faculty and doctoral level when compared to the combined ethnic diversity in all engineering disciplines (Figures 2 b, c). The ethnic diversity of the Asian/Pacific Islander group at the chemical engineering undergraduate level is favorable when compared to the overall engineering population. It is evident that a larger number of students from the Asian/Pacific Islander group move on to get doctoral degrees - this group constitutes about $15 \%$ of the chemical engineering undergraduate population and $31.25 \%$ of chemical engineering doctoral student population. However, the level of diversity of the same group at the faculty level drops to $21.9 \%$ and to $14 \%$ at the Chemical Engineering division membership level (Figures 2 $\mathrm{a}$-c). Thus there is a need to recruit and retain more chemical engineering faculty members and Chemical Engineering division members from the Asian/Pacific Islander group.

Diversity of black-non-Hispanic and Hispanic students graduating from chemical engineering undergraduate programs is less than the combined ethnic diversity in all engineering disciplines. Thus there is a need to recruit and retain more students from these groups in chemical engineering undergraduate programs to have an ethnic diversity that is commensurate with the overall engineering population (Figure $2 \mathrm{~d}$ ). In general the ethnic diversity pipeline has bottlenecks at the undergraduate, doctoral, and faculty levels. (Figures $2 \mathrm{a}-\mathrm{d}$ ). The percentage of black-non-Hispanic and Hispanic members in the Chemical Engineering division matches their proportion in the chemical engineering professorship. These two groups are heavily underrepresented compared to the general working population and efforts should be taken to recruit and retain people from black-non-Hispanic and Hispanic groups at all levels of chemical engineering education.

The American Indian / Alaskan native groups are the most underrepresented group - to an extent that their population in the engineering disciplines is usually in fractional percentages. Since this is the least represented group, serious efforts needs to be made to recruit people from this group at all levels of chemical engineering education. 


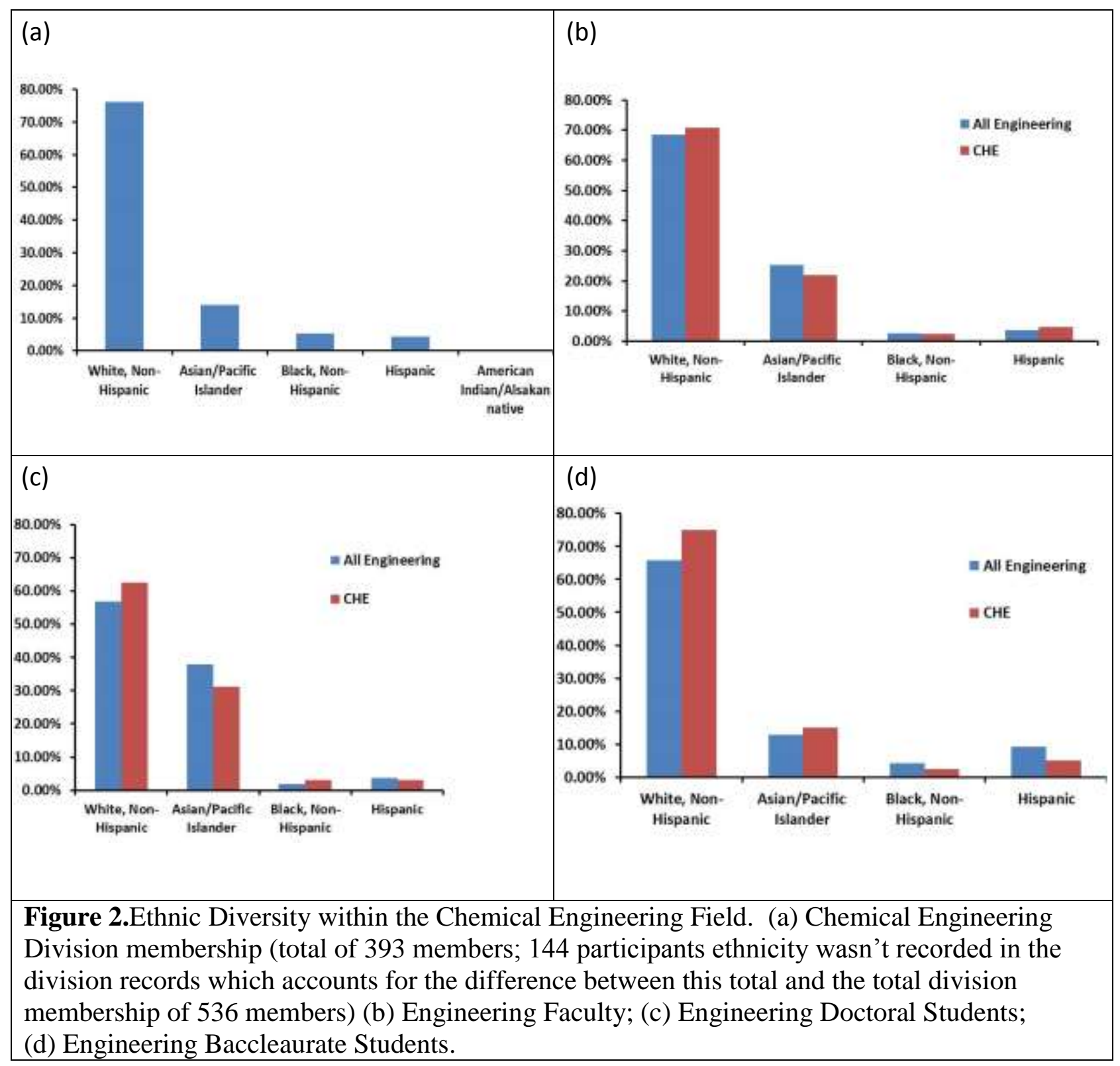

\section{Faculty Perspectives on Diversity in Chemical Engineering}

As part of the committee's goal to inform the chemical engineering education division on the state of diversity and perspectives on this issue, the division will host a panel as part of this year's ASEE annual meeting in conjunction with the Women in Engineering Division. The panel consists of six panelists' representative of a variety of different academic positions and a broad range of institutions within the United States. Each of the panelists for the panel was selected based on their professional background and passion for working towards a more inclusive population within the chemical engineering field.

In preparation for the upcoming panel, the panelists were given a six question survey that allowed them to weigh in their thoughts on the current state of diversity within the profession, challenges that lie ahead and what needs to be done if we would like to attain the goals that have 
been set not only as a profession but within the broader engineering field to create a diverse talent pool for tackling the grand challenges that lie ahead. The following section will summarize the themes that were noted in the panelists' responses and lay the foundation for the discussion that will take part during the panel session.

As mentioned briefly the panelists in this panel are representative of different positions within the academic environment as well as diverse administrative responsibilities. Represented on the panel are a lecturer, assistant professor, associate professor, professor of practice and two full professors. The administrative responsibilities of the panelists vary with their institution but include assistant chair to the department, undergraduate advising, graduate student advising, assisting with University Strategic Planning and Budget, Associate Dean for Undergraduate Education and Interim Dean of the College of Engineering. The institutions that were represented by the panelists selected included four R1 research institutions with the other two being considered to have a high research focus. There was also a split in the nature of the institutions with four of them falling under public funded universities and two being private.

The first question that panelists were asked was to describe how their institutions foster an inclusive environment where differences are celebrated and valued. In response to this the panelists shared examples of diversity offices in place that had both faculty and staff members to help support an inclusive environment, the provision of academic support programs, such as EXCEL, to help with student success, summer bridging programs to aid in a better transition to the university environment and inclusion of mentoring programs. It was also routinely discussed how some of the support does not come from academic programs alone but the existence of student clubs and programs that celebrate everyone's differences and hence encourage an environment of inclusivity. Although it was clear from their responses that many programs are in place to help encourage both recruitment and retention of a diverse student and faculty body it was mentioned that additional resources were necessary to help support these existing programs and also encourage growth in these areas over the years ahead.

Panelists were then asked to discuss whether based on their experiences any gender or racial biases might manifest within the academic workplace and how these could be addressed. It was saddening but perhaps not surprising to note that every panelist on the panel had noted some level of gender or racial bias throughout their careers. One of the recurring themes is that these biases were very subtle and not necessarily easy to identify. Inherently everyone does have some level of implicit bias but in most cases we are not trained to be able to identify these biases within ourselves which can make them difficult to correct.

Some of the specific examples of bias that were raised were having more women as instructors for first and second year courses to help with retention of under-represented populations which would prevent them from having the opportunity to teach more senior level courses, perceived roles that men/women are supposed to adopt within a work setting resulting in negative consequences when these are not met, and attributing successes these individuals have to their gender or race rather than due to their personal scientific merits. Another common practice was placement of under-represented minorities on departmental committees to assure diversity even though they were typically the only under-represented individual included. All of these subtle biases can lead to an environment that is the opposite of what we are trying to create. Instead of 
having under-represented individuals feel included and celebrated for the diverse perspectives that they can contribute they are led to feeling isolated like imposters. This can also place them in situations where they don't feel they can voice their opinions such as being the only underrepresented minority present on a committee. The long term impact of these biases results in a decrease in productivity that can impact the ability they have to reach key milestones such as tenure or receipt of awards.

Another area of discussion was best practices for faculty hiring, retention and promotion. It was noted that each of these areas should be addressed separately with their own specific resources. To ensure that we are capable of recruiting and hiring more individuals from these populations it is necessary that there be a broader marketing strategy when performing faculty searches. This should include reaching out to specific colleges and institutions that might have larger populations of under-represented minorities either within their graduate programs, post-doctoral programs or within their non-tenure track positions. It also involves inviting individuals from these populations on to campus before the search process even starts to familiarize them with the opportunities that may be available to them in a faculty position at that institution. Search committee members should be provided with proper training to allow for identification of implicit bias that might impact their search process. As mentioned earlier, these biases exist whether we are aware of them or not but when we identify them we can better mitigate their presence. Once the pool of faculty candidates has been expanded to include more individuals from the under-represented population it is helpful to consider whether there is the possibility of a cluster hire rather than just a single hire. Through a cluster hire, where more than one underrepresented minority is hired at one time, it is possible to create a community that would provide the support necessary to help success once the individual starts their position. It also helps to address the issues that have been noted with opportunity hires where the under-represented individuals may not have the necessary support to achieve long term impact once they start in their positions.

Better retention and the ability to improve the number of individuals receiving promotion both contributed to the environment that is created within the institution. Provision of a community, network or mentorship opportunities and professional development training were all felt to be extremely helpful in generating an environment for future success. Mentoring could be provided internally but also externally through professional organizations. Resources at the campus level were also found to be helpful in creating programs that could provide this support network whether through funding received from the NSF ADVANCE program or through other diversity specific initiatives. It was also discussed that ensuring there are open lines of communication with these new faculty members and letting them know that they are free to seek assistance and help when needed will encourage their development and success. Finally, the concept of sponsorship and the need for advocates for under-represented minorities was also raised. It was believed that without the availability of individuals who are willing to serve as sponsors, we would continue to see fewer under-represented individuals both reaching full professor status and being promoted into leadership positions.

When panelists were asked what the top three challenges for enhancing diversity in engineering education in general and in particular within chemical engineering were they identified them as (1) Pipeline, (2) Supportive Environment, and (3) Resource Limitations. The pipeline was 
considered to be especially crucial since this is the starting point for being able to recruit diverse populations into engineering. It was also noted by one panelist that there tends to be a feedforward effect within the K-12 space as the career based surveys administered in our high schools define populations that belong in those careers based on the current workforce population. However, by perpetuating this trend through the use of the current workforce as models for future students we will not be able to achieve the change we would like to see as we have not yet reached a diverse workforce within engineering to base these surveys upon. Other pipeline issues that remain to be addressed included the public perception of engineering and how we could attract more people to this field as well as being able to tie engineering to some of the other fields such as health care, global issues and social justice that have historically been shown to be more appealing to an under-represented population.

In an effort to provide a more supportive environment for under-represented minorities several suggestions were raised by the panelists. It was stated that diversity is an issue that affects all of us and hence these discussions should not only take place amongst the under-represented populations but as a broader population within the chemical engineering field. If we truly want to generate change in the environment we will need to ensure a collective change toward inclusiveness and support from all stakeholders. Other suggestions that were provided included having more representation of under-represented individuals within the faculty and allowing for non-traditional career pathways.

The final challenge that was outlined by panelists was resource limitations. Although funding organizations are doing their best to try to support programs and create new ones that could lead to a more diverse engineering workforce, oftentimes these funds are not sufficient for long term sustainability of these goals. It will be important for institutions to also invest additional time and resources to help support the work that is initiated through external funding and come up with creative solutions to ensure their propagation after the end of the initial funding period.

The last question that was posed to panelists was how we as a community could work together to reduce/mitigate gender and racial biases. Common themes that emerged included bringing broader awareness to the issue, providing training about these biases and approaches that could help mitigate them and generating open discussion on the subject. It was felt that this year's "ASEE Year of Action on Diversity" is a good first step to bringing this issue to the forefront.

The key takeaway message from the faculty perspectives discussed is that diversity is an issue that should be important to all of us. If we truly want to be able to tackle the engineering projects of the future we need to have a trained workforce that encompasses multiple perspectives and can work together fluidly to bring ideas to fruition. As one panelist stated "Being open and inclusive of differences, and viewing those as assets means that all possible solutions are evaluated equally based on merits and the truly best engineered solution is attained."

\section{Conclusions and Future Directions}

Diversity of our profession is very important for several reasons including the need to generate creative and diverse approaches to the expanding nature of problems within the chemical 
engineering field. Through research that was performed it was observed that the number of publications relevant to the fields of diversity is still at a very small number within the premier engineering education journals of the United States and Europe (Journal of Engineering Education and European Journal of Engineering Education specifically). It is of utmost importance that diversity related studies should continue to be published and promoted within these journals due to the high value placed upon them within the engineering education field. This will not only help raise awareness of the issue but also promote best practices for institutions and faculty members to follow to ensure an inclusive environment.

Review of the gender and ethnicity distribution within the Chemical Engineering division and the field provided insight on the current status within this field. It was found that although diversity could still be improved at all levels to be more representative of the general working population, the division has been doing reasonably well at encouraging participation within the division by both women and under-represented minorities. At the time of this review, it was found that particular effort still needs to be made to encourage broader participation of the Asian/Pacific Islander group so that there is a more equal representation of this group within the division in comparison to the field of chemical engineering.

Perceptions from faculty members on diversity demonstrated that we still have issues that need to be addressed before we are able to generate inclusive environments. The three main challenges that they identified included pipeline issues, generation of a supportive environment and resource limitations for diversity related programming. Although many institutions have made significant steps forward through creation of diversity offices, cluster hires and mentoring programs there is work that still remains to be done. All of the faculty members felt that we can change diversity within the profession but it can only be done when we all come together as a community to discuss diversity issues and brainstorm new solutions that haven't yet been tried.

The future directions for the Chemical Engineering division diversity committee include utilizing the panel session at this year's ASEE annual meeting as a starting point for discussion with the larger chemical engineering community. The Diversity Initiatives Committee will work with the Chemical Engineering Education editorial board to publish a diversity special issue in the near future. This issue will provide the opportunity to showcase the excellent work that is being done by educators within the chemical engineering field to address diversity related issues and help bring better prominence to this central issue within the field as a whole.

\section{References}

1. Wulf, W.A. (1998). Diversity in Engineering. The Bridge. 28(4).

2. NSF (2013). Women, Minorities, and Persons with Disabilities in Science and Engineering. http://www.nsf.gov/statistics/wmpd/2013/digest/theme4.cfm. Last Accessed January 30, 2015.

3. Engineering by The numbers (2013). http://www.asee.org/papers-and-publications/publications/14_1147.pdf. Last accessed January $30^{\text {th }}, 2015$.

4. ASEE (2015). Diversity. http://diversity.asee.org/awards Accessed on January 31, 2015.

5. Manicom, T (2013). Personal Communications 